\title{
Commensurate and incommensurate states of topological quantum matter
}

\author{
Ashley Milsted, ${ }^{1}$ Emilio Cobanera, ${ }^{2, *}$ Michele Burrello, ${ }^{3}$ and Gerardo Ortiz ${ }^{4}$ \\ ${ }^{1}$ Institut für Theoretische Physik, Leibniz Universitat Hannover, Appelstrasse 2, D-30167 Hannover, Germany \\ ${ }^{2}$ Instituut-Lorentz, Universiteit Leiden, P.O. Box 9506, NL-2300 RA Leiden, The Netherlands \\ ${ }^{3}$ Max-Planck-Institut für Quantenoptik, Hans-Kopfermann-Strasse 1, D-85748 Garching, Germany \\ ${ }^{4}$ Department of Physics, Indiana University, Bloomington, Indiana 47405, USA
}

(Received 3 July 2014; revised manuscript received 4 September 2014; published 3 November 2014)

\begin{abstract}
We prove numerically and by dualities the existence of modulated, commensurate and incommensurate states of topological quantum matter in systems of parafermions, motivated by recent proposals for the realization of such systems in mesoscopic arrays. In two space dimensions, we obtain the simplest representative of a topological universality class that we call Lifshitz. It is characterized by a topological tricritical point where a nonlocally ordered homogeneous phase meets a disordered phase and a third phase that displays modulations of a nonlocal order parameter.
\end{abstract}

DOI: 10.1103/PhysRevB.90.195101

PACS number(s): $05.30 . \mathrm{Rt}, 11.15 . \mathrm{Ha}, 75.10 . \mathrm{Kt}$

\section{INTRODUCTION}

In recent years, most efforts directed at investigating topological quantum matter experimentally have taken a top-to-bottom approach, starting from a model Hamiltonian and engineering a system to realize it. From this point of view, mesoscopic superconducting arrays have already been proven successful [1], and also for cold atomic gases the implementation of topological phases of matter seems within reach [2].

Inevitably, the model Hamiltonians in question can only be realized up to implementation-dependent modifications, which, although small, may still be relevant in the sense of the renormalization group and drive large systems away from the intended topological phase. This practical aspect of the theory of phase transitions for topological quantum matter is the natural counterpart of analogous considerations for conventional systems like magnetic memories, which can only tolerate some range of temperatures and applied magnetic fields. However, there is one crucial difference. Since a Landau theory of nonlocal order parameters, which are those appropriate for topological quantum matter, does not exist yet, it is very difficult to predict and classify interacting topological gapless phases. By contrast, the classification of gapped phases is well developed (for parafermions, see Refs. [3,4]).

In this paper we extend the list of demonstrated topological critical behaviors (see, for example, Refs. [5-10]). We show that topological quantum matter can be driven into phases characterized by nonlocal orders incommensurate with the underlying lattice. Remarkably, it will become clear that modulated and floating (and, in particular, incommensurate) topological quantum orders can easily arise in mesoscopic arrays from very natural interactions. And we prove the existence of a topological universality class surprisingly sensitive to an underlying lattice structure by locating a topological Lifshitz tricritical point in the phase diagram of a two-dimensional model of topological quantum matter.

Let us recall the basics of modulated Landau orders. When a local order parameter $\Phi(\boldsymbol{x})$ emerges in a lattice system,

\footnotetext{
*cobanera@lorentz.leidenuniv.nl
}

phases may occur in which this order parameter displays modulations $\Phi(\boldsymbol{x}) \sim \Phi_{0} \cos \left(\boldsymbol{k}_{0} \cdot \boldsymbol{x}+\phi_{0}\right)$ commensurate with the lattice periodicity. The wave vector $\boldsymbol{k}_{0}$ is restricted by the Lifshitz condition to take one of a few possible values in the first Brillouin zone [11].

This picture of modulated local orders can break down if interactions that favor competing periodicities are present, as exemplified by the anisotropic next-nearest-neighbor Ising (ANNNI) model [12,13] of magnetic ordering in the heavy lanthanoids [14]. In systems with such competing interactions, there might be regimes where the equilibrium wave vector varies continuously with some driving force, as first predicted in Ref. [15] from the Landau functional density

$$
f=\kappa_{2} \Phi^{2}+\kappa_{4} \Phi^{4}+\kappa_{6} \Phi^{6}+\gamma_{1}(\nabla \Phi)^{2}+\gamma_{2}\left(\nabla^{2} \Phi\right)^{2}
$$

for an Ising order parameter. Just as the standard Ising tricritical point emerges at $\kappa_{2}=\kappa_{4}=0$, the Lifshitz tricritical point emerges at $\gamma_{1}=0$. It is the coexistence point for the paramagnetic, ferromagnetic, and modulated phases of the local order parameter $\Phi$. On the coexistence line between the modulated and the paramagnetic phases, starting at the Lifshitz point, the wave vector $\boldsymbol{k}$ varies continuously with the driving field and so an additional critical exponent appears. If $\boldsymbol{k}$ happens to vary continuously in some (regime of some) phase, then the regime/phase is called floating.

In the following we demonstrate through explicit examples that the full range of phenomena associated with commensurate and incommensurate modulations and the Lifshitz point can also be present in topological quantum matter, but now in terms of nonlocal order parameters. Unlike the situation for local (Landau) orders just discussed, there is no obvious way to predict such topological quantum orders on the basis of some general Landau-Wilson functional. This point showcases one of the troubling limitations in our current understanding of topological quantum matter at criticality.

\section{A ONE-DIMENSIONAL PHYSICAL REALIZATION}

We start by considering a one-dimensional effective Hamiltonian with a discrete global $\mathbb{Z}_{2 m}(m=1,3, \ldots$, odd $)$ symmetry that displays a critical floating regime. One may obtain a $\mathbb{Z}_{2 m}$ symmetry in systems with quasiparticles of fractional 


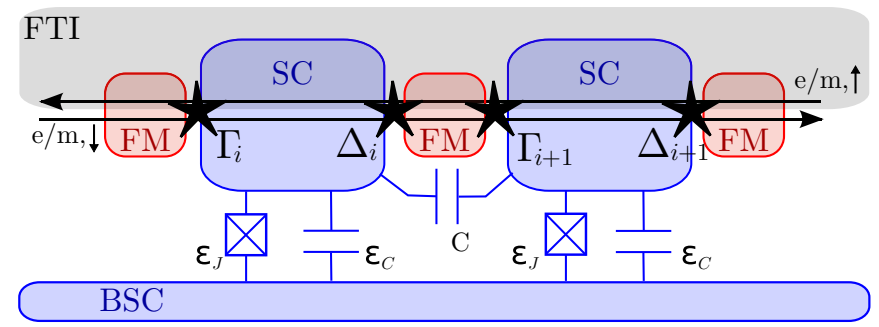

FIG. 1. (Color online) Parafermions $\Gamma_{i}$ and $\Delta_{i}$ are localized along the edge of an FTI at the interfaces between superconducting islands (SC) and insulating ferromagnets (FM). Each superconducting island is coupled to neighboring islands via a capacitive coupling $C$ and to a ground superconductor (BSC) via a Josephson junction $\varepsilon_{J}$ and a capacitive coupling $\varepsilon_{C}$.

charge $e / m$ subjected to proximity-induced superconducting pairing. The combination of these two ingredients provides a channel for Cooper pairs to split into $2 m$ indistinguishable parts. Then the condensation of the Cooper pairs leads to a peculiar cyclic behavior of the local, charged degrees of freedom and induces the required $\mathbb{Z}_{2 m}$ symmetry. These ideas are central to several proposals [16-19] that aim to realize localized parafermionic zero-energy modes (parafermions, for short) in hybrid mesoscopic arrays including fractional topological insulators (FTIs). Parafermions are obtained by gapping the edge modes of an FTI and constitute a fractionalized version of Majorana zero-energy edge modes (Majoranas, for short), allowing for the emergence of one-dimensional systems which generalize [3,4,10,20,21] the well-known Majorana-Kitaev chain [22].

Along the edge of an FTI, localized parafermions emerge at the interfaces between alternating regions where the edge modes of the FTI are gapped by proximity to superconducting islands or insulating ferromagnets [16,17] (see Fig. 1). Each superconducting island $i$ hosts a pair of parafermionic modes $\Gamma_{i}, \Delta_{i}$ sharing a fractional charge $q_{i}^{\mathrm{f}}=0, \frac{1}{m}, \frac{2}{m}, \ldots, \frac{2 m-1}{m}$, in units of $e$, defined modulo 2 [16,17]. Parafermions obey nonlocal commutation rules,

$$
\begin{gathered}
\Gamma_{i} \Delta_{j}=e^{i \frac{\pi}{m}} \Delta_{j} \Gamma_{i} \quad(i \leqslant j), \\
\Gamma_{i} \Gamma_{j}=e^{i \frac{\pi}{m}} \Gamma_{j} \Gamma_{i}, \quad \Delta_{i} \Delta_{j}=e^{i \frac{\pi}{m}} \Delta_{j} \Delta_{i} \quad(i<j), \\
\Gamma_{i}^{2 m}=\mathbb{1}=-\Delta_{i}^{2 m}, \quad \Gamma_{i} \Gamma_{i}^{\dagger}=\Delta_{i} \Delta_{i}^{\dagger}=\mathbb{1} .
\end{gathered}
$$

This algebra of parafermions is a natural generalization of the Clifford algebra of Majoranas.

The charge $q_{i}^{\mathrm{f}}$ is the charge of the FTI edge segment coupled to the superconductor and may be represented by the operator $\Gamma_{i}^{\dagger} \Delta_{i}=e^{i \pi q_{i}^{\mathrm{f}}}$. In our mesoscopic array, two main physical processes intervene to couple the zero-energy modes: a fractional Josephson effect [17,19], which generalizes the electron tunneling mediated by Majoranas [23]; and the charging interactions of the islands, which, just as in the Majorana case [24-26], cause an energy splitting of the states with different fractional charges [27]. The Josephson interaction accounts for the tunneling of fractional quasiparticles between two neighboring islands and it changes their fermionic number by $\pm 1 / m$. The tunneling of a single fractional charge is the dominant process, and in terms of parafermionic modes, it reads $-\left(E_{J} / 2\right)\left(\Gamma_{i+1} \Delta_{i}^{\dagger}+\right.$ H.c. $)$. The charging interactions are modeled by assuming that each island is coupled to a background superconductor by a strong normal Josephson junction and a capacitive contact, with magnitudes $\varepsilon_{J}$ and $\varepsilon_{C}$, respectively. See Fig. 1.

Besides the contribution coming from Cooper pairs, the total charge in each island includes the charge $q_{i}^{\text {ind }}$ induced by the neighboring potentials and the fractional charge $q_{i}^{\mathrm{f}}$ associated with the parafermions. The effect of these two contributions is especially important if $\varepsilon_{J} \gg \varepsilon_{C}$, that is, in the transmon regime [28]. In this regime the low-energy physics can be described by semiclassically assuming that the superconducting phase of the island is approximately pinned to the minima of the Josephson energy. Then the charging energy causes an effective interaction $-\Delta_{C} \cos \left[\pi\left(q_{i}^{\mathrm{f}}+q_{i}^{\text {ind }}\right)\right]$, where $\Delta_{C}$ depends on the ratio $\varepsilon_{J} / \varepsilon_{C}$ [28], and the cosine dependence is due to the Aharonov-Casher effect associated with $2 \pi$-phase slips in states with different charges $q_{i}^{\mathrm{f}}+$ $q_{i}^{\text {ind }}$ [24,26]. Following Ref. [27], this interaction may be written as $-(1 / 2)\left(E_{C}^{(1)} \Gamma_{i}^{\dagger} \Delta_{i}+\right.$ H.c. $)$, where $E_{C}^{(1)}=\Delta_{C} e^{-i \pi q^{\text {ind }}}$ is, in general, complex. It is possible to tune $q^{\text {ind }}$, using voltage gates in the system, to take the value 0 or 1 and thus obtain a positive or negative single-island charging energy term.

A further charging term appears in the presence of a cross-capacitance $C$ between neighboring islands. This term originates from the simultaneous $2 \pi$-phase slip of both islands [26] and reads $-E_{C}^{(2)} \cos \left[\pi\left(q_{i}^{\mathrm{f}}+q_{i+1}^{\mathrm{f}}+q_{i}^{\text {ind }}+q_{i+1}^{\text {ind }}\right)\right]$. In particular, we impose that all the induced charges share a common value $q^{\text {ind }}$. By tuning $q^{\text {ind }}$ to add a unit of charge to each island $\left(q_{i}^{\text {ind }} \rightarrow q_{i}^{\text {ind }}+1\right)$, the relative sign between the coupling strengths $E_{C}^{(1)}$ and $E_{C}^{(2)}=\left|E_{C}^{(2)}\right| e^{-i 2 \pi q_{\text {ind }}}$ may be controlled. This cross-capacitance interaction is translated into a four-parafermion operator, and combining all the previous terms, we obtain an effective Hamiltonian,

$$
\begin{aligned}
H_{\mathrm{eff}}= & -\frac{1}{2} \sum_{i=1}^{L}\left[\left(E_{J} \Gamma_{i+1} \Delta_{i}^{\dagger}+E_{C}^{(1)} \Gamma_{i}^{\dagger} \Delta_{i}\right.\right. \\
& \left.\left.+E_{C}^{(2)} \Gamma_{i}^{\dagger} \Delta_{i} \Gamma_{i+1}^{\dagger} \Delta_{i+1}\right)+ \text { H.c. }\right]
\end{aligned}
$$

for the description of the array in Fig. 1 in its low-energy sector with periodic boundary conditions. In the following, we take $E_{C}^{(1)}=1$ and $E_{C}^{(2)} \leqslant 0$. Then $H_{\text {eff }}$ is closely connected to a generalization of the ANNNI model (corresponding to $m=1$ ) to any odd $m$ (see the Appendix).

\section{Quantum phase diagram: Commensurate and incommensurate topological phases}

We studied the quantum phase diagram of $H_{\text {eff }}$ for $m=3$ numerically, computing approximate ground states using the open source evoMPS toolbox [29], which implements variational tangent plane techniques for matrix product states (MPSs) [30]. In particular, evoMPS implements the nonlinear conjugate gradient method to accelerate the process significantly, particularly for critical regimes, in comparison to imaginary time evolution [31]. We choose block 


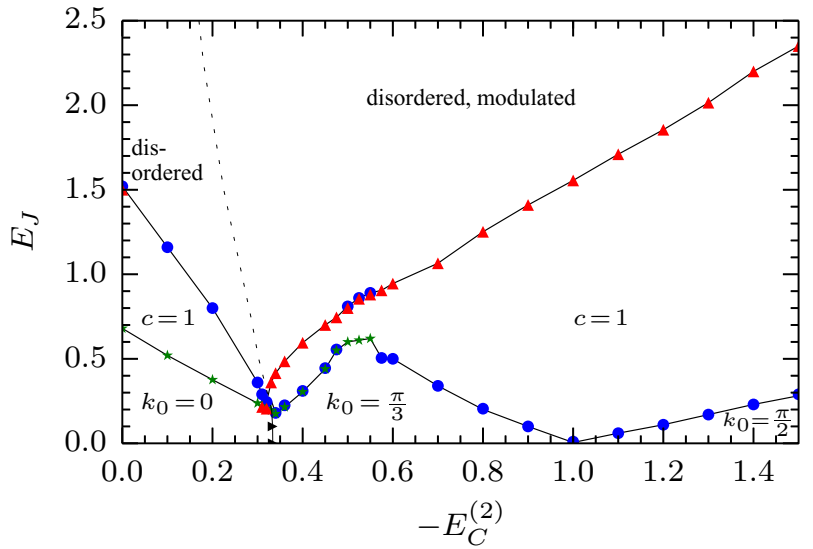

FIG. 2. (Color online) Quantum phase diagram of $H_{\text {eff }}$ for $m=$ 3. The three ordered phases are labeled by the wave vector associated with the modulation of the string order parameter. The disordered phase contains modulated and unmodulated regimes, separated by the (dashed) disorder line. There are two critical phases with central charge $c=1$. Various indicators were used to mark the transitions: (blue) circles mark global maxima of the entanglement entropy, (green) stars mark local maxima of its first $E_{J}$ derivative, (red) upward triangles mark local minima of the second $E_{J}$ derivative of the ground energy, and black rightward triangles mark discontinuities in the first derivative of the ground energy.

translation-invariant MPSs with various block lengths in order to handle ground states with nontrivial periodicity.

The quantum phase diagram of $H_{\text {eff }}$ is shown in Fig. 2. Three gapped phases appear at low $E_{J} \geqslant 0$ and present a topologically protected ground-state degeneracy, corresponding, in finite-size systems, to the total fractional charge. These gapped phases are followed by two critical phases, both with central charge $c=1$ [32], which, in turn, are topped by a gapped phase at large $E_{J}$, showing no ground-state degeneracy. To further characterize the (dis)orders in these phases, we follow the ideas of Refs. [33] and [34] to determine a nonlocal order parameter by mapping $H_{\text {eff }}$ to a Landau-ordered system. We obtain (see Appendix) that the ground-state $|\Omega\rangle$ expectation value

$$
\Sigma_{i}(d)=\left\langle\Omega\left|\prod_{n=i}^{i-d+1} \Gamma_{n}^{\dagger} \Delta_{n}\right| \Omega\right\rangle
$$

(independent of $i$ ) defines the required nonlocal order parameter. $\Sigma_{i}(d)$ displays long-range order in the three phases at small $E_{J}$, with modulations characterized by $k_{0}=0, \pi / 3, \pi / 2$. The wave vectors are ordered as they appear for increasing $-E_{C}^{(2)}$ (Fig. 2). The ordered phases with $k_{0}=0, \pi / 3$ are separated by a first-order line.

Starting at $E_{J}=0$ in the gapped phase with either $k_{0}=\pi / 3$ or $k_{0}=\pi / 2$ and increasing $E_{J}$ along a vertical line, the system enters the critical phase at the right in Fig. 2, and the asymptotic behavior of $\Sigma_{i}(d)$ changes from long ranged to algebraically decaying, but with a modulation $k_{0}\left(E_{J}\right)$ that appears to vary continuously with $E_{J}$ to the best available computer resolution. In this regime, the periodicity of the nonlocal order in the system is no longer anchored to the lattice structure, so our mesoscopic array demonstrates the existence of floating

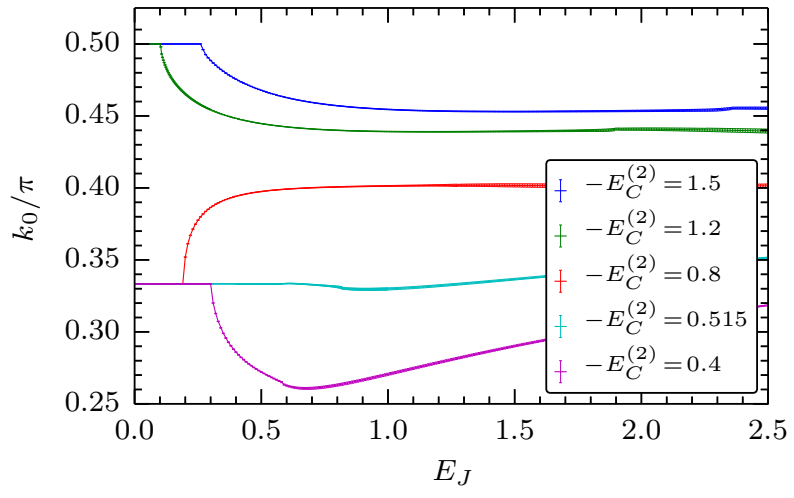

FIG. 3. (Color online) Fitting of $\operatorname{Re}\left[\Sigma_{i}(d)\right]$ with a decay function modulated with wave vector $k_{0}$. The wave vectors start at a constant value in the ordered phases and change continuously in the the critical phase at the right of the phase diagram. Error bars represent statistical errors from the least-squares fitting.

regimes for mesoscopically realized topological quantum matter. Figure 3 shows $k_{0}\left(E_{J}\right)$ for the full range of $E_{J}$ for three values of $-E_{C}^{(2)}$ starting at $k_{0}=\pi / 3$ and two values starting at $k_{0}=\pi / 2$.

As for the other phases, there are no modulations in the critical phase at the left of the phase diagram. At $-E_{2}^{(C)}=0$, this phase is precisely (see the Appendix) the critical phase of the $p=2 m=6$ clock model (see Ref. [35] and references therein). The modulations of the string order parameter survive in the the gapped, disordered phase at large $E_{J}$, where $\Sigma_{i}(d)$ decays exponentially rapidly in $d$, but only for sufficiently large values of $-E_{C}^{(2)}$. There is a regime in the disordered phase without modulations, as shown in Fig. 2. The separation between the two disordered regimes, unmodulated and modulated, is called the disorder line in the literature on the ANNNI model.

The string-ordered phases of $H_{\text {eff }}$ manifest the various ways in which the global, discrete symmetry,

$$
U_{\mathrm{C}}=\prod_{i=1}^{L} \Gamma_{i}^{\dagger} \Delta_{i}, \quad U_{\mathrm{C}}^{2 m}=\mathbb{1}, \quad\left[U_{\mathrm{C}}, H_{\mathrm{eff}}\right]=0,
$$

can be spontaneously broken in the limit of infinite system size. There are, however, topological quantum orders that emerge without spontaneously breaking any symmetries, as first noticed for Ising gauge theories [36]. These states of topologically quantum matter are often modeled by systems with local symmetries, since, by Elitzur's theorem [37], local symmetries cannot be spontaneously broken. The remainder of the paper focuses on a model that displays incommensurate behavior and even a full-fledged topological Lifshitz point, without spontaneous symmetry breaking. We call a Lifshitz point topological if it is a tricritical point of the Lifshitz type but associated only with nonlocal orders.

\section{TOPOLOGICAL LIFSHITZ UNIVERSALITY CLASS}

The model in question, inspired by the mesoscopic realization of the toric code in terms of Majoranas [38], features parafermions $\Gamma_{(\boldsymbol{r}, \mu)}, \Delta_{(\boldsymbol{r}, \mu)}(\mu=1,2)$ on each link 
$(\boldsymbol{r}, \mu)$ connecting sites $\boldsymbol{r}, \boldsymbol{r}+\boldsymbol{e}_{\boldsymbol{\mu}}$ of a square lattice. Let us define plaquette operators $B_{\boldsymbol{r}}=U_{(\boldsymbol{r}, 1)} U_{\left(\boldsymbol{r}+\boldsymbol{e}_{1}, 2\right)} U_{\left(\boldsymbol{r}+\boldsymbol{e}_{2}, 1\right)}^{\dagger} U_{(\boldsymbol{r}, 2)}^{\dagger}$ [in terms of the shorthand notation $U_{(\boldsymbol{r}, \mu)}=\Gamma_{(\boldsymbol{r}, \mu)}^{\dagger} \Delta_{(\boldsymbol{r}, \mu)}$ and star operators $\left.A_{\boldsymbol{r}}=\Delta_{(\boldsymbol{r}, 1)} \Gamma_{\left(\boldsymbol{r}-\boldsymbol{e}_{2}, 2\right)}^{\dagger} \Delta_{(\boldsymbol{r}, 2)} \Gamma_{\left(\boldsymbol{r}-\boldsymbol{e}_{1}, 1\right)}^{\dagger}\right]$. As the naming suggests, the star and plaquette operators generate a commutative algebra. The gapped Hamiltonian

$$
H_{\mathrm{TC}}=-\frac{1}{2} \sum_{r}\left[h_{p} B_{r}+h_{s} A_{r}+\text { H.c. }\right]
$$

is precisely the parafermionic representation of the $\mathbb{Z}_{2 m}$ toric code. In the following we study the effect of the perturbation

$$
\begin{aligned}
V= & -\frac{J_{1}}{2} \sum_{\boldsymbol{r}, \mu}\left[U_{(\boldsymbol{r}, \mu)}+\text { H.c. }\right] \\
& -\frac{J_{2}}{2} \sum_{\boldsymbol{r}}\left[U_{(\boldsymbol{r}, 2)} U_{\left(\boldsymbol{r}+\boldsymbol{e}_{1}, 2\right)}+\text { H.c. }\right],
\end{aligned}
$$

with $J_{1},-J_{2} \geqslant 0$. Since the plaquette operators $B_{r}$ commute with the full Hamiltonian $H_{\mathrm{LTC}}=H_{\mathrm{TC}}+V$, they play the role of local symmetries. The ground state of the system belongs to the gauge-invariant sector where the plaquettes $B_{r}$ act as the identity.

For the purpose of realizing the topological Lifshitz universality class, it suffices to consider only the simplest case of $m=1$ for which the parafermions reduce to Majoranas. Following [21], we exploit a gauge-reducing duality transformation [39] to map $H_{\text {LTC }}$ to a dual Landau-ordered system $H_{\text {LTC }}^{D}$. Because we fix $m=1$, this dual system features spins $S=1 / 2$ placed at the sites $\boldsymbol{r}$ of a square lattice, represented by Pauli matrices $\sigma_{r}^{\alpha}$. It is governed by the Hamiltonian

$$
\begin{aligned}
H_{\mathrm{LTC}}^{D}= & -\sum_{\boldsymbol{r}}\left(h_{s} \sigma_{\boldsymbol{r}}^{x}+h_{p} \mathbb{1}\right)-J_{1} \sum_{\boldsymbol{r}, \mu} \sigma_{\boldsymbol{r}}^{z} \sigma_{\boldsymbol{r}-\boldsymbol{e}_{\mu}}^{z} \\
& -J_{2} \sum_{\boldsymbol{r}} \sigma_{\boldsymbol{r}-\boldsymbol{e}_{\boldsymbol{1}}}^{z} \sigma_{\boldsymbol{r}+\boldsymbol{e}_{\boldsymbol{1}}}^{z}
\end{aligned}
$$

The dual Hamiltonian $H_{\mathrm{LTC}}^{D}$ is precisely the celebrated quantum ANNNI model in two space dimensions. In the mean field approximation, $H_{\mathrm{LTC}}^{D}$ is directly connected to the Landau functional of Eq. (1) [13]. Since dualities are unitary transformations [39], we obtain that our perturbed toric code and the ANNNI model share identical phase diagrams. In the following we rely on the extensive knowledge of this phase diagram collected in Ref. [13].

To characterize the nonlocal (dis)orders in the quantum phase diagram as it pertains to the topological model $H_{\mathrm{LTC}}$, we need to identify a nonlocal order parameter. Again, we follow the ideas of Ref. [33] and obtain (see the Appendix) the string order parameter,

$$
\Sigma_{\boldsymbol{r}}(d)=\left\langle\Omega\left|\prod_{j=1}^{d} U_{\left(\boldsymbol{r}+j \boldsymbol{e}_{1}, 2\right)}\right| \Omega\right\rangle .
$$

In terms of $h_{s}$ vs $-J_{2} / J_{1}$, the phase diagram splits into a phase at high $h_{s}$ with exponential decay of $\Sigma_{r}(d)$ and phases at low $h_{s}$ with long-range string order. The ordered phases are split by a phase boundary starting at $-J_{2} / J_{1}=0.5, h_{s}=0$ into a homogeneous phase $k_{0}=0$ at low $-J_{2} / J_{1}$ and a modulated phase for stronger $-J_{2}$, composed of many (possibly infinitely many) modulated phases with various $k_{0} \neq 0$. The two types of string orders meet the string disordered phase at a topological Lifshitz point. In other words, our model Hamiltonian $H_{\text {LTC }}$ realizes the topological Lifshitz universality class.

\section{CONCLUSIONS}

In summary, we have proved that competing interactions in topological systems can lead to commensurate and incommensurate nonlocal orders with distinct critical behaviors, without affecting the ground-state degeneracy of the topological gapped phases. There are clear directions for future research. On the experimental side, it may be easier to demonstrate incommensurate nonlocal orders in cold atoms [40] rather than mesoscopic arrays, and so it would be interesting to investigate models presenting modulated phases for the string order parameter associated with the Haldane phase of $S=1$ spin chains. On the theoretical side, it is possible that the topic of modulated topological quantum orders opens an area of research significantly wider in scope than its Landau counterpart. To ascertain whether this is the case it would help to characterize the interplay between modulated orders and gauge fields. A natural starting point would be to investigate, in terms of the Fredenhagen-Marcu string order parameter recently rederived from dualities [33], the phase diagram of a $\mathbb{Z}_{2}$ Higgs model with the matter field controlled by the ANNNI model Hamiltonian.

\section{ACKNOWLEDGMENTS}

We thank B. van Heck, Y. Nakata, and L. Vanderstraeten for useful discussions. A.M. was supported by the ERC Grants QFTCMPS and SIQS and by the cluster of excellence EXC 201 Quantum Engineering and Space-Time Research. E.C. was supported by the Dutch Science Foundation NWO/FOM and an ERC Advanced Investigator grant. M.B. acknowledges support from the Cluster of Excellence EXC 4 Nanosystems Initiative Munich and from the EU Grant SIQS.

\section{APPENDIX}

Duality transformations. We report here the duality transformations mentioned in the paper, following closely the techniques introduced in Refs. [33,39].

For the Hamiltonian $H_{\text {eff }}$, the duality transformation in question is the unitary transformation $\mathcal{U}_{\mathrm{d}}$ induced by the mapping of interactions

$$
\Gamma_{i}^{\dagger} \Delta_{i} \mapsto \Delta_{i}^{\dagger} \Gamma_{i+1}, \quad \Delta_{i}^{\dagger} \Gamma_{i+1} \mapsto \Gamma_{i+1}^{\dagger} \Delta_{i+1} \quad(i=1, \ldots, L)
$$

The isospectral dual Hamiltonian $H_{\text {eff }}^{D}=\mathcal{U}_{\mathrm{d}} H_{\text {eff }} \mathcal{U}_{\mathrm{d}}^{\dagger}$ reads

$$
\begin{aligned}
H_{\mathrm{eff}}^{D}= & -\frac{1}{2} \sum_{i=1}^{L}\left[\left(E_{J} \Gamma_{i}^{\dagger} \Delta_{i}+E_{C}^{(1)} \Delta_{i}^{\dagger} \Gamma_{i+1}\right.\right. \\
& \left.\left.+E_{C}^{(2)} \Delta_{i}^{\dagger} \Gamma_{i+1} \Delta_{i+1}^{\dagger} \Gamma_{i+2}\right)+ \text { H.c. }\right] .
\end{aligned}
$$


It is useful to rewrite $H_{\text {eff }}^{D}$ in terms of local degrees of freedom. The combinations

$$
U_{i}=\Gamma_{i}^{\dagger} \Delta_{i}, \quad V_{i}=\Gamma_{i} \prod_{m=1}^{i-1} \Delta_{m}^{\dagger} \Gamma_{m},
$$

of parafermions define spinlike, so-called clock variables that commute on different sites, and otherwise satisfy

$$
V_{i} U_{i}=e^{i \frac{\pi}{m}} U_{i} V_{i}, \quad U_{i}^{2 m}=U_{i} U_{i}^{\dagger}=\mathbb{1}=V_{i} V_{i}^{\dagger}=V_{i}^{2 m} .
$$

For $m=1$, these relations are satisfied by letting $U_{i} \rightarrow \sigma_{i}^{z}$ and $V_{i} \rightarrow \sigma_{i}^{x}$, with $\sigma_{i}^{x}, \sigma_{i}^{z}$ the standard Pauli matrices. Then the reciprocal relations

$$
\Gamma_{i}=V_{i} \prod_{m=1}^{i-1} U_{m}, \quad \Delta_{i}=\Gamma_{i} U_{i}
$$

show that, for $m=1, \Gamma_{i} \rightarrow a_{i}$ and $\Delta_{i} \rightarrow-i b_{i}$, with $a_{i}, b_{i}$ standard Majorana fermions satisfying the standard relation $c_{i}=\left(a_{i}+i b_{i}\right) / 2$ to ordinary fermions.

In terms of the local clock variables $U_{i}, V_{i}$, and up to boundary terms that we neglect in the following, $H_{\text {eff }}^{D}$ reduces to

$$
\begin{aligned}
& H_{\mathrm{ANNNC}} \\
& \quad=-\frac{1}{2} \sum_{i}\left[E_{J} U_{i}+E_{C}^{(1)} V_{i}^{\dagger} V_{i+1}+E_{C}^{(2)} V_{i}^{\dagger} V_{i+2}+\text { H.c. }\right] .
\end{aligned}
$$

For $E_{C}^{(2)}=0$, the Hamiltonian $H_{\mathrm{ANNNC}}$ reduces to the standard clock model [35,39]. For $E_{C}^{(2)}<0, H_{\mathrm{ANNNC}}$ describes a ferromagnetic clock model with antiferromagnetic next-nearestneighbor interactions. For $m=1$, the clock variables are just Pauli matrices and $H_{\mathrm{ANNNC}}$ becomes the quantum descendant of the two-dimensional classical ANNNI model [13]. Hence we call $H_{\text {ANNNC }}$ the anisotropic next-nearest-neighbor clock (ANNNC) model. The phases of the ANNNC model can be distinguished by the long-distance behavior of the two-point correlator $g_{i}(d)=\left\langle V_{i}^{\dagger} V_{i+d}\right\rangle$. This observation translates into the string order parameter

$$
\Sigma_{i}(d)=\left\langle\Omega\left|\prod_{n=i}^{i-d+1} \Gamma_{n}^{\dagger} \Delta_{n}\right| \Omega\right\rangle
$$

for $H_{\text {eff }}$, by applying the transformations just introduced to $g_{i}(d)$.

For the two-dimensional Hamiltonian $H_{\mathrm{LTC}}=H_{\mathrm{TC}}+V$ (see Fig. 4 for an illustration of the notation), and $m=1$, the duality mapping reads

$$
\begin{aligned}
A_{\boldsymbol{r}} & \mapsto \sigma_{\boldsymbol{r}}^{x}, \\
U_{(\boldsymbol{r}, 1)} & \mapsto \eta_{(\boldsymbol{r}, 1)} \sigma_{r}^{z} \sigma_{r-e_{2}}^{z}, \\
U_{(\boldsymbol{r}, 2)} & \mapsto \eta_{(\boldsymbol{r}, 2)} \sigma_{r}^{z} \sigma_{r-e_{1}}^{z} .
\end{aligned}
$$

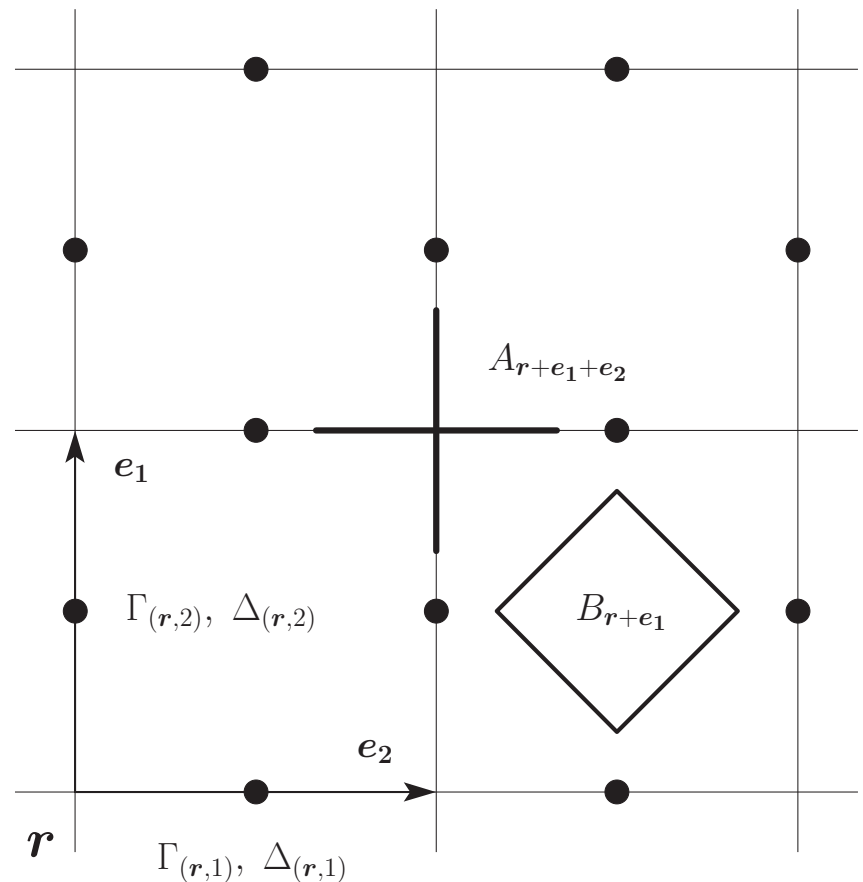

FIG. 4. Parafermions for the two-dimensional systems $H_{\text {LTC }}$.

The classical Ising variables $\eta_{\boldsymbol{r}, \mu}= \pm 1$ are fixed in accordance with the relation

$$
B_{\boldsymbol{r}} \mapsto\left(\prod_{\mu=1}^{2} \eta_{(\boldsymbol{r}, \mu)} \eta_{\left(\boldsymbol{r}-\boldsymbol{e}_{\mu}, \mu\right)}\right) \mathbb{1}
$$

so that the dual system represents our perturbed toric code projected onto a particular set of simultaneous eigenstates of the $B_{r}$. The gauge-invariant sector corresponds to $\eta_{(\boldsymbol{r}, \mu)}=1$. Magnetic phases that are distinguished in the ANNNI model $H^{D}$ by the long-distance behavior of the two-point correlator

$$
g_{r}(d)=\left\langle\sigma_{r}^{z} \sigma_{r+d e_{1}}^{z}\right\rangle
$$

are distinguished in our perturbed toric code by the string correlator

$$
\Sigma_{\boldsymbol{r}}(d)=\left\langle\Omega\left|\prod_{i=1}^{d} U_{\left(\boldsymbol{r}+m e_{1}, 2\right)}\right| \Omega\right\rangle .
$$

Numerical methods. To compute the phase diagram and wave vectors presented in this work, we first obtain approximate ground states of $H_{\text {eff }}$ or $H_{\text {eff }}^{D}$ using block translation invariant MPS

$$
|\Psi[A]\rangle=\sum_{\vec{s}=0}^{p} v_{L}^{\dagger}\left[\prod_{n=-\infty}^{+\infty} A_{0}^{s_{n L}} \cdots A_{L-1}^{s_{(n+1) L-1}}\right] v_{R}|\vec{s}\rangle,
$$

where $A_{k}^{s}$ is a $D \times D$ complex matrix or parameters, $D$ is the bond dimension, $\vec{s}=s_{-\infty} \cdots s_{+\infty}$, and $v_{L}, v_{R}$ are boundary vectors that do not feature in our calculations since the bulk is completely decoupled from the infinitely distant boundaries. To obtain a well-defined norm and expectation values, we also require that the transfer matrix $E=\sum_{s_{0} \cdots s_{L-1}} A_{0}^{s_{0}} \cdots A_{L-1}^{s_{L-1}} \otimes$ 
$\overline{A_{0}^{S_{0}} \cdots A_{L-1}^{S_{L-1}}}$ has a unique eigenvalue of largest magnitude equal to one.

By exploiting the tangent space $\mathcal{T}_{[A]}[30]$ to the variational manifold $\mathcal{M}_{D}$ defined in (A14) at a given bond dimension $D$, it is possible to compute the effective energy gradient (imaginary time evolution), which can be used to implement the nonlinear conjugate gradient method for minimizing the energy [31]. The tangent plane consists of vectors $\partial_{i}|\Psi[A]\rangle$, where $i$ enumerates all entries in the set of tensors $[A]$. These methods, among others, are implemented in the open source Python package evoMPS [29].

To obtain the phase diagram in the main text, we fix $D$, in this case to $D=16$ or $D=24$, and compute MPS ground states along lines in parameter space, sweeping in both possible directions and selecting the lowest energy state for each point. We begin with a block length of $L=1$, increasing it if it becomes clear that the energy minimization is leading towards a global superposition (in order to restore translation invariance), which is indicated by the appearance of multiple eigenvalues of $E$ with magnitude approximately equal to one. We use a variety of quantities to locate a probable transition, in particular the first and second ground-state energy derivatives, the entanglement entropy and correlation length, the expectation value of the order parameter, and its correlation function. We test for criticality within a region by computing an estimate for the CFT central charge from the scaling of the entropy and the correlation length with the bond dimension [32]. Note that, to precisely locate and characterize a second order (or higher order) phase transition, the bond dimension should be increased until finite entanglement effects are no longer significant.

We estimate the wave vector of the correlation function modulation by fitting the correlation function (or string expectation value) over 20 sites using

$$
f(d)=A e^{-d \lambda} \cos \left(k_{0} d+\phi\right),
$$

where $d$ is the distance in sites, $\lambda$ is the inverse correlation length, $\phi$ is an offset, and $k_{0}$ is the wave vector. We obtain an error on $k_{0}$ from the least squares fit result. Although the decay is approximately algebraic (for short distances) within critical regions, this function still offers a good fit of the wave vector. Within a modulated critical region, the wave vector is also present as the phase of the second largest eigenvalue of $E$, the magnitude of which determines the correlation length [30].

For this work, we used ground-state data for both $H_{\text {eff }}^{D}$ and $H_{\text {eff }}$, finding the results to be consistent. $H_{\text {eff }}^{D}$ offers some numerical advantages, possessing only nearest-neighbor interactions and having typically smaller ground-state periodicity.
[1] S. Gladchenko, D. Olaya, E. Dupont-Ferrier, B. Doucot, L. B. Ioffe, and M. E. Gershenson, Nat. Phys. 5, 48 (2009).

[2] N. Goldman, G. Juzeliunas, P. Ohberg, and I. B. Spielman, arXiv:1308.6533.

[3] R. Bondesan and T. Quella, J. Stat. Mech. (2013) P10024.

[4] J. Motruk, E. Berg, A. M. Turner, and F. Pollmann, Phys. Rev. B 88, 085115 (2013).

[5] E. Ardonne, P. Fendley, and E. Fradkin, Ann. Phys. 310, 493 (2004).

[6] A. Feiguin, S. Trebst, A. W. W. Ludwig, M. Troyer, A. Kitaev, Z. Wang, and M. H. Freedman, Phys. Rev. Lett. 98, 160409 (2007).

[7] I. S. Tupitsyn, A. Kitaev, N. V. Prokofev, and P. C. E. Stamp, Phys. Rev. B 82, 085114 (2010).

[8] S. Dusuel, M. Kamfor, R. Orus, K. P. Schmidt, and J. Vidal, Phys. Rev. Lett. 106, 107203 (2011).

[9] M. D. Schulz, S. Dusuel, R. Orus, J. Vidal, and K. P. Schmidt, New J. Phys. 14, 025005 (2012).

[10] W. Li, S. Yang, H.-H. Tu, and M. Cheng, arXiv:1407.3790.

[11] L. D. Landau and E. M. Lifshitz, Statistical Physics, 2nd ed. (Pergamon Press, New York, 1968), Chap. XIV.

[12] P. Bak, Rep. Prog. Phys. 45, 587 (1982).

[13] W. Selke, Phys. Rep. 170, 213 (1988).

[14] R. J. Elliott, Phys. Rev. 124, 346 (1961).

[15] R. M. Hornreich, M. Luban, and S. Shtrikman, Phys. Rev. Lett. 35, 1678 (1975).

[16] N. H. Lindner, E. Berg, G. Refael, and A. Stern, Phys. Rev. X 2, 041002 (2012).

[17] D. J. Clarke, J. Alicea, and K. Shtengel, Nat. Commun. 4, 1348 (2013).
[18] A. Vaezi, Phys. Rev. B 87, 035132 (2013).

[19] M. Cheng, Phys. Rev. B 86, 195126 (2012).

[20] P. Fendley, J. Stat. Mech. (2012) P11020.

[21] E. Cobanera and G. Ortiz, Phys. Rev. A 89, 012328 (2014).

[22] A. Y. Kitaev, Phys. Usp. 44, 131 (2001).

[23] C. Xu and L. Fu, Phys. Rev. B 81, 134435 (2010).

[24] B. van Heck, F. Hassler, A. R. Akhmerov, and C. W. J. Beenakker, Phys. Rev. B 84, 180502 (2011).

[25] B. van Heck, A. R. Akhmerov, F. Hassler, M. Burrello, and C. W. J. Beenakker, New J. Phys. 14, 035019 (2012).

[26] F. Hassler and D. Schuricht, New J. Phys. 14, 125018 (2012).

[27] M. Burrello, B. van Heck, and E. Cobanera, Phys. Rev. B 87, 195422 (2013). This paper uses the notation $\alpha_{i}$ to denote parafermions. The correspondence with our notation is $\alpha_{2 i}=\Gamma_{i}$ and $\alpha_{2 i+1}=e^{-i \frac{\pi}{2 m}} \Delta_{i}$.

[28] J. Koch, T. M. Yu, J. Gambetta, A. A. Houck, D. I. Schuster, J. Majer, A. Blais, M. H. Devoret, S. M. Girvin, and R. J. Schoelkopf, Phys. Rev. A 76, 042319 (2007).

[29] evoMPS source, http://amilsted.github.io/evoMPS/

[30] J. Haegeman, T. J. Osborne, and F. Verstraete, Phys. Rev. B 88, 075133 (2013); A. Milsted, J. Haegeman, T. J. Osborne, and F. Verstraete, ibid. 88, 155116 (2013).

[31] A. Milsted, J. Haegeman, and T. J. Osborne, Phys. Rev. D 88, 085030 (2013).

[32] L. Tagliacozzo, T. R. de Oliveira, S. Iblisdir, and J. I. Latorre, Phys. Rev. B 78, 024410 (2008); V. Stojevic, J. Haegeman, I. P. McCulloch, L. Tagliacozzo, and F. Verstraete, arXiv:1401.7654.

[33] E. Cobanera, G. Ortiz, and Z. Nussinov, Phys. Rev. B 87, 041105(R) (2013). 
[34] B. van Heck, E. Cobanera, J. Ulrich, and F. Hassler, Phys. Rev. B 89, 165416 (2014).

[35] G. Ortiz, E. Cobanera, and Z. Nussinov, Nucl. Phys. B 854, 780 (2012).

[36] F. Wegner, J. Math. Phys. 12, 2259 (1971).

[37] S. Elitzur, Phys. Rev. D 12, 3978 (1975).
[38] B. M. Terhal, F. Hassler, and D. P. Di Vincenzo, Phys. Rev. Lett. 108, 260504 (2012).

[39] E. Cobanera, G. Ortiz, and Z. Nussinov, Adv. Phys. 60, 679 (2011); Phys. Rev. Lett. 104, 020402 (2010).

[40] M. Endres et al., Science 334, 200 (2011). 